\title{
GANGLIONEUROMA SUPRARRENAL. APORTACIÓN DE UN NUEVO CASO
}

\author{
F. ARREDONDO MARTÍNEZ, M. SOTO DELGADO, A. BENAVENTE FERNÁNDEZ*, \\ B. BASQUERO GONZÁLEZ, A. ZURERA COSANO, R. LINARES ARMADA
}

Servicio de Urología. *Servicio de Medicina Interna. Hospital Infanta Elena. Huelva.

Actas Urol Esp. 27 (3): 221-225, 2003

\section{RESUMEN}

"GANGLIONEUROMA SUPRARRENAL. APORTACIÓN DE UN NUEVO CASO"

El ganglioneuroma es una neoplasia benigna derivada de la cresta neural, compuesta de células ganglionares maduras y células de Schwann. La mayoría se localizan en el mediastino posterior, seguido del retroperitoneo. Sólo una pequeña proporción de ganglioneuromas se originan en la médula suprarrenal, siendo más frecuentes en niños y adultos jóvenes.

Característicamente, los ganglioneuromas no secretan catecolaminas ni hormonas esteroideas, por lo que suelen ser lesiones clínicamente silentes que son detectadas incidentalmente en estudios de imagen realizados por otros motivos no relacionados.

Por ser lesiones infrecuentes, los ganglioneuromas no suelen ser incluidos en el diagnóstico diferencial de los incidentalomas suprarrenales, particularmente en adultos.

Presentamos un nuevo caso de ganglioneuroma suprarrenal de $6,5 \mathrm{~cm}$, diagnosticado incidentalmente en un paciente de 50 años de edad. La exéresis de dicha masa y su posterior estudio histopatológico confirmaron el diagnóstico.

PALABRAS CLAVE: Ganglioneuroma. Glándula suprarrenal. Incidentaloma. Edad adulta.

\section{ABSTRACT \\ "ADRENAL GANGLIONEUROMA. TO REPORT OF A NEW CASE"}

Ganglioneuroma is a benign neoplasm arising from neural crest tissue and is composed of mature ganglion cells and Schwann's cells. Most ganglioneuromas arise in the posterior mediastinum followed by the retroperitoneum. Only a small proportion of ganglioneuromas are adrenal in origin and occur most commonly in children and young adults.

Characteristically, ganglioneuromas do not secrete excess catecholamines or steroid hormones, and they are usually clinically silent lesions incidentally detected in imaging studies for unrelated reasons.

Because it is an uncommon lesion, adrenal ganglioneuromas is not included in the differential diagnosis of adrenal incidentalomas in adults.

We presents a new case of a $6.5 \mathrm{~cm}$ adrenal ganglioneuroma incidentally diagnosed in a 50year old male patient. The exeresis of the adrenal mass and pathohistological study confirmed the diagnosis.

KEY WORDS: Ganglioneuroma. Adrenal gland. Incidentaloma. Adults. 
$\mathrm{E}^{\mathrm{s}}$ ganglioneuroma es una neoplasia benigna derivada de la cresta neural, compuesta de células ganglionares maduras y células de Schwann. Pueden surgir en cualquier lugar a lo largo del plexo simpático paravertebral, siendo su lugar de asiento más frecuente el mediastino posterior, seguido del retroperitoneo, particularmente el espacio presacro. Sólo una pequeña proporción de ganglioneuromas se originan en la médula suprarrenal ${ }^{1,2}$.

Aunque pueden ocurrir en todos los grupos de edad, la mayoría se presentan en pacientes menores de 20 años ${ }^{1}$.

Los ganglioneuromas carecen de secreción hormonal, por lo que suelen comportarse como lesiones clínicamente silentes que son detectadas de forma incidental en estudios de imagen realizados por otras razones.

A pesar de que el ganglioneuroma es una neoplasia bien conocida, no suele incluirse en el diagnóstico diferencial de las masas suprarrenales incidentales en el adulto, debido a su excepcional presentación en estas edades.

Presentamos un caso de ganglioneuroma suprarrenal derecho detectado incidentalmente y tratado quirúrgicamente en un varón de 50 años.

\section{CASO CLÍNICO}

Paciente varón de 50 años de edad, sin antecedentes personales ni familiares de interés, remitido a consulta externa de urología ante el hallazgo en ecografía abdominal, realizada por síntomas digestivos inespecíficos, de una tumoración suprarrenal derecha sólida de $6 \mathrm{~cm}$ de diámetro.

La anamnesis y la exploración física no revelaron sintomas ni signos que orientaran hacia la presencia de una endocrinopatía, ni hacia la existencia de un tumor primario.

Se procedió, mediante estudio endocrinológico, a establecer el carácter funcionante o no de la tumoración suprarrenal. Se determinaron: cortisol libre en orina; cortisol en orina de 24 horas; supresión con $1 \mathrm{mgr}$ de dexametasona; cortisol basal en sangre; ACTH; aldosterona en sangre; DHEA-sulfato; catecolaminas en sangre (noradrenalina, adrenalina) y en orina, y sus metabolitos (vanilmandélico, metanefrinas). Los resultados de los parámetros analíticos practicados fueron todos normales.
El TAC abdominal demuestra la presencia de una masa suprarrenal derecha sólida, de contornos bien definidos y densidad heterogénea, con áreas hipodensas e imágenes de densidad calcio en su interior, que mide $6,5 \times 3 \mathrm{~cm}$ (Fig. 1). La Resonancia Magnética muestra una masa ovalada y bien definida, de $6,5 \mathrm{~cm}$ de diámetro mayor, isointensa respecto al bazo en $\mathrm{T} 1$ y T2, con áreas irregulares hiperintensas en T2 (Figs. 2 y 3).

El estudio concluyó la presencia de una masa suprarrenal derecha sólida, de $6,5 \mathrm{~cm}$ de diámetro $\mathrm{y}$ no funcionante. Con este diagnóstico, y apoyados fundamentalmente en el tamaño de la tumoración, así como en la presencia de calcificaciones irregulares en su interior, se decidió la exéresis quirúrgica de la misma ante la posibilidad de que se tratara de un carcinoma suprarrenal. Se practicó suprarrenalectomía derecha a través de lumbotomía.

Macroscópicamente, la pieza de suprarrenalectomía midió $8 \times 6,5 \times 3 \mathrm{~cm}$. La tumoración muestra una superficie multinodular, constituida por un tejido homogéneo, blanquecino-grisáceo de aspecto fasciculado. Microscópicamente, el tumor está constituido por células de Schwann dispuestas en pequeños fascículos entrelazados con presencia de focos de estroma mixoide y grupos de células ganglionares maduras. Se realizó estudio inmunohistoquímico para determinación de proteína S-100, observándose intensa y generalizada inmunoreacción en las células neoplásicas. El diagnóstico definitivo es de ganglioneuroma suprarrenal (Fig. 4).



FIGURA 1. Masa suprarrenal derecha sólida, ovalada, que mide $6,5 \times 3 \mathrm{~cm}$, heterogénea y con pequeñas calcificaciones en su interior. 

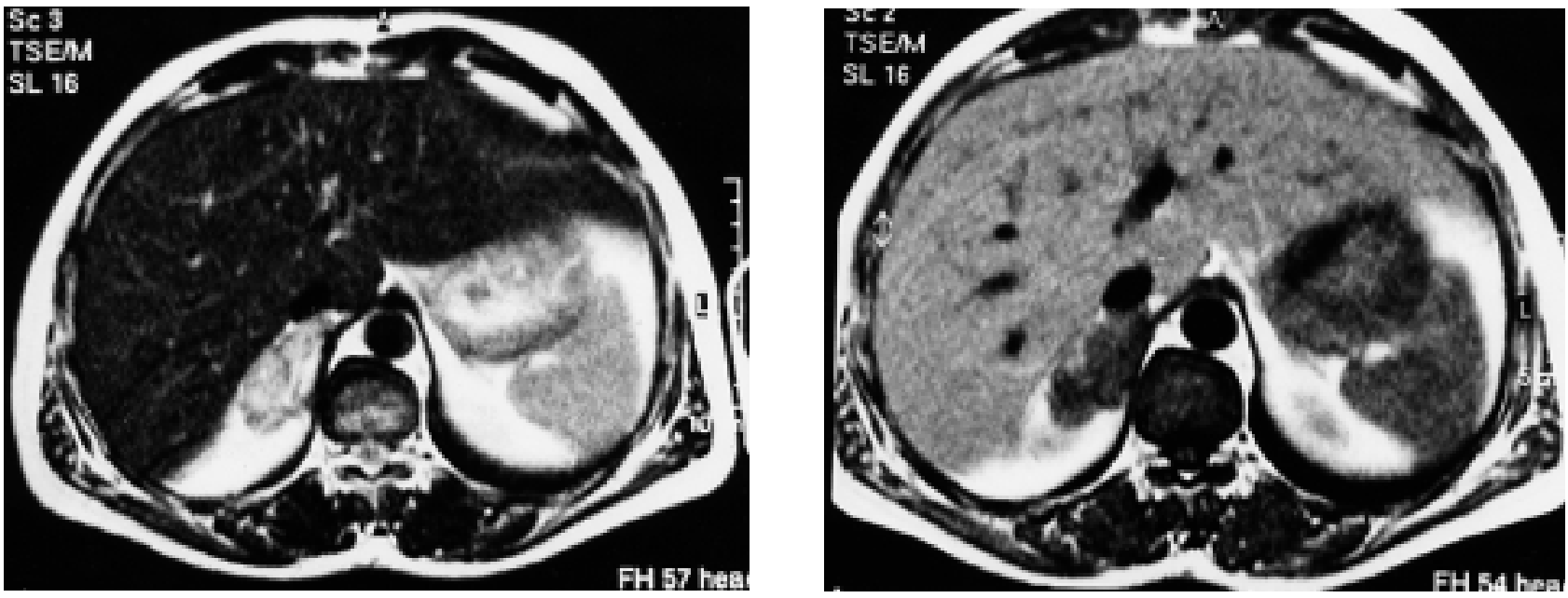

FIGURAS 2 y 3. Masa ovalada y bien definida de 6,5 cm, isointensa respecto al bazo en T1 y T2 con áreas irregulares hiperintensas en $\mathrm{T} 2$.

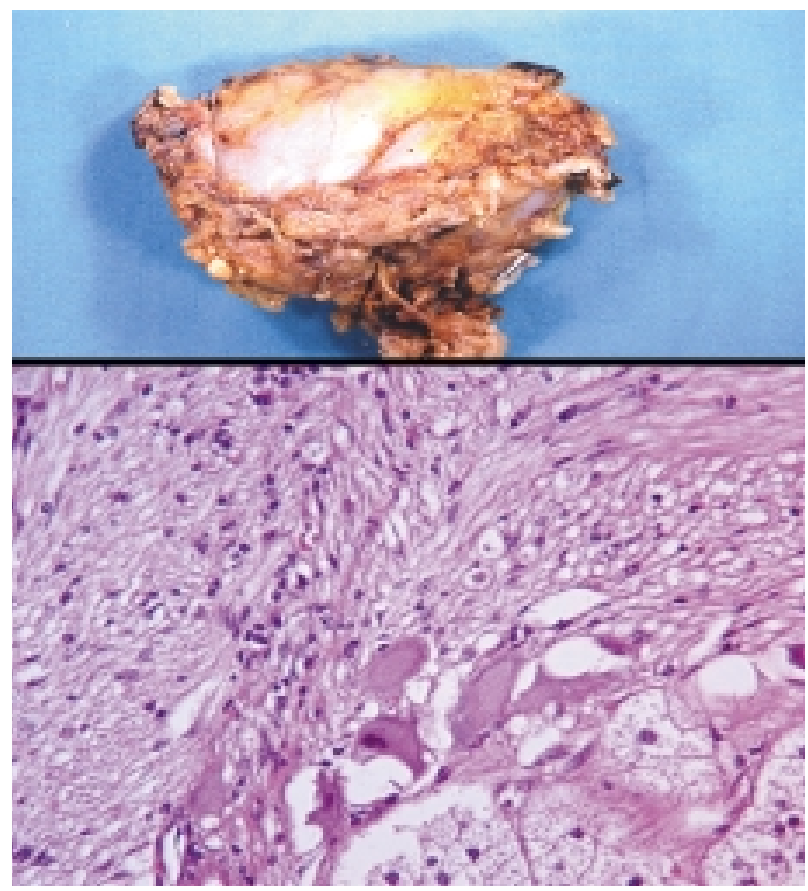

FIGURA 4. Macro y micro (HE 40x): en la porción periférica de la pieza se pueden ver entremezcladas células ganglionares, células pertenecientes a la cortical adrenal y células de Schwann.

Tras 2 años de seguimiento el paciente se encuentra asintomático y sin evidencias de recidiva de la lesión.

\section{DISCUSIÓN}

Los tumores originados en la cresta neural incluyen un espectro de tumores benignos y malignos. Se originan a partir de las neuronas simpáticas primitivas de la cresta neural, que son el origen de la médula suprarrenal y de los ganglios simpáticos. El neuroblastoma es el más frecuente, siendo menos habituales el ganglioneuroblastoma y el ganglioneuroma ${ }^{1-3}$.

El ganglioneuroma es el tumor más infrecuente derivado de la cresta neural. Es un tumor completamente diferenciado que no contiene elementos inmaduros, formado por células ganglionares maduras y células de Schwann en un estroma fibroso, representando el estadío final de diferenciación de las células neuroblásticas de la cresta neural. Existe controversia sobre si los ganglioneuromas se originan de novo o por maduración o diferenciación a partir de un neuroblastoma o un ganglioneuroblastoma preexistente. Sin embargo, las diferencias tanto en la edad de presentación como en la localización, apoyan la idea de que los ganglioneuromas no necesariamente se desarrollan a partir de un neuroblastoma preexistente, surgiendo la mayoría de novo ${ }^{4}$.

Macroscópicamente, son tumores bien circunscritos y encapsulados. Su tamaño es variable, con un promedio de $8 \mathrm{~cm}$, aunque se han descrito casos de hasta $5 \mathrm{Kg}^{5}$.

La mayoría se localizan en el mediastino posterior, seguido del retroperitoneo, particularmente en el espacio presacro. Solamente el 15-30\% de los casos notificados asientan en la glándula suprarrenal. Otras localizaciones descritas son: cuello, área parafaríngea, vejiga urinaria, próstata y apéndice ${ }^{1,10}$. 
Aunque el ganglioneuroma puede ocurrir en cualquier grupo de edad, más del $80 \%$ están descritos en pacientes menores de 40 años, y de éstos más de la mitad son menores de 20 años 6 .

Para la mayoría de los autores los ganglioneuromas son tumores benignos. De los 147 tumores estudiados por Stout ${ }^{7}$, ninguno metastatizó. Sin embargo, se han notificado varios casos de transformación maligna de ganglioneuroma hacia Schwanoma maligno ${ }^{8,9}$. También pueden ocurrir en asociación con otros tumores como el feocromocitoma $^{11}$.

Característicamente, el ganglioneuroma no es un tumor secretor de catecolaminas ni de hormonas esteroideas. Suelen presentarse como lesiones clínicamente silentes que son diagnosticadas de forma incidental en estudios de imagen realizados por otros motivos ${ }^{2,6}$. Ocasionalmente se asocian a diarrea, debido a la liberación de péptido intestinal vasoactivo, sudoración e hipertensión ${ }^{12}$. Así mismo se ha descrito un caso en el contexto de un cuadro de miastenia gravis y virilización $^{13}$. El síntoma no endocrinológico más frecuente es el dolor lumbar crónico inespecífico.

El diagnóstico diferencial en el adulto, habrá que realizarlo con otras lesiones suprarrenales descubiertas incidentalmente. Aunque la mayoría de los "incidentalomas"suprarrenales son benignos (adenomas, mielolipomas, quistes...), y el carcinoma suprarrenal primario es excepcional, la posibilidad de que éste exista determina la necesidad de su identificación y tratamiento.

Además, hay que investigar masas funcionantes sin clínica aparente. Esto hace necesario una valoración hormonal básica ${ }^{14}$. La demostración de hiperfunción indicará generalmente la necesidad de cirugía.

Un pequeño porcentaje de tumores suprarrenales no funcionantes presentan características en la TAC o la RMN que permiten establecer un diagnóstico preciso (mielolipoma, hemorragia aguda, quiste) ${ }^{15,16}$. El ganglioneuroma se muestra en el TAC como una lesión sólida, homogénea con una atenuación más baja que el músculo. Algunos son heterogéneos tras la administración de contraste, pero la mayoría, tienden a permanecer menos densos que el músculo. Pueden presentar pequeñas calcificaciones hasta en un $20 \%$ de los casos, siendo otra característica interesante su tendencia a envolver total o parcialmente los vasos sanguíneos sin comprometer su luz. La RMN los presenta como lesiones con una señal homogéneamente baja en T1, y una señal heterogénea, predominante alta en T2. Sin embargo, el ganglioneuroma no ofrece características discriminatorias que permitan establecer un diagnóstico seguro ${ }^{6}$.

El diagnóstico definitivo lo establece el estudio histológico de la pieza quirúrgica. La punciónaspiración con aguja fina (PAAF), representa una alternativa, aunque sus indicaciones se han restringido (sospecha de metástasis y lesiones quísticas) debido a la dificultad para distinguir entre adenoma y carcinoma bien diferenciado ${ }^{17}$.

En el caso de tumores sólidos no funcionantes, la decisión de exéresis quirúrgica la establece fundamentalmente el tamaño de la lesión, debiendo ser extirpadas aquellas mayores de $6 \mathrm{~cm}$. Más controvertida es la actitud a seguir cuando son menores de $6 \mathrm{~cm}$, ayudando en estos casos determinadas características radiológicas (TAC, RMN) o gammagráficas, si bien el seguimiento clínico es otra alternativa aceptada, optando por la cirugía si el tamaño de la lesión se modifica ${ }^{18-20}$.

En nuestro caso se optó por la extirpación quirúrgica de la lesión, apoyándonos fundamentalmente en el tamaño de la misma $(6,5 \mathrm{~cm})$, así como por la presencia de calcificaciones irregulares en su interior, ante la posibilidad de que se tratara de un carcinoma suprarrenal.

\section{REFERENCIAS}

1. ENZINGER FM, WEISS SW.: Soft tissue tumors. $3^{\text {rd }}$ ed. St Louis, Mo: Mosby-Year Book 1995: 929-964.

2. SCHULMAN H, LAUFER L, BARKI Y, PHILIP M et al.: Ganglioneuroma: an "incidentaloma" of childhood. Eur Radiol 1998; 8: 582-584.

3. RODRÍGUEZ J, LANCINA JA, GÓMEZ F y cols.: Tumores retroperitoneales derivados de la cresta neural. Revisión de conjunto. Act Urol Esp 1992; 16: 516.

4. ENZINGER FM, WEISS SW.: Soft tissue tumors. St Louis: CV Mosby 1988: 828-831.

5. CARPENTER WB, KERNOHAN JW.: Retroperitoneal ganglioneuromas and neurofibromas. A clinicopathological study. Cancer 1963; 16: 788-797.

6. RANDALL R, CYNTHIA LD, HELENE G, ISAAC RF.: Adrenal and extra-adrenal retroperitoneal ganglioneuroma: imaging findings in 13 adults. Radiology 1997; 202: 703-707.

7. STOUT AP.: Ganglioneuroma of the sympathetic nervous system. Surg Gynecol Obstet 1947; 84: 101-110. 
8. FLETCHER CD, FERNANDO IN, BRAINBRIDGE MV, MCKEE PH, LYALL JR.: Malignant nerve sheath tumor arising in a ganglioneuroma. Histopathology 1988; 12: 445-448.

9. RICCI AJ, CALLIHAN T, PARHAM DM, GREEN A et al.: Malignant peripheral nerve sheath tumors arising from ganglioneuromas. Am J Surg Pathol 1984; 8: 19-29.

10. ERNEST E, LACK MD.: Tumors of the adrenal gland and extra-adrenal paraganglia: Atlas of tumor pathology. Third Series. Fascicle 19, 1995.

11. CHETTY R, DUHIG JD.: Bilateral pheochromocytoma-ganglioneuroma of the adrenal in type 1 neurofibromatosis. Am J Surg Pathol 1993; 17: 837841.

12. HANSEN LP, LUND HT, FAHRENKRUG J, SOGAARD H.: Vasoactive intestinal polypeptide (VIP)producing ganglioneuroma in a child with chronic diarrhea. Acta Pediatr Scand 1980; 69: 419424.

13. AGUIRRE P, SCULLY RE.: Testosterone-secreting adrenal ganglioneuroma containing Leydig cells. Am J Surg Pathol 1983; 7: 699-705.

14. ROSS NS, ARON DC.: Hormonal evaluation of the patient with an incidentally discovered adrenal mass. N Engl J Med 1990; 323: 1401-1405.
15. FRANCIS IR, GROSS MD, SHAPIRO B et al.: Integrated imaging of adrenal disease. Radiology 1992; 184: 1.

16. KOROBKIN M, BRODEUR FJ, YUTZY GG et al.: Differentiation of adrenal adenomas from nonadenomas using CT attenuation values. AJR Am J Roentgenol 1996; 166: 531.

17. KLOOS RT, GROSS MD, FRANCIS IR et al.: Incidentally discovered adrenal mass. Endocr Rev 1995; 16: 460.

18. COPELAND PM.: The incidentally discovered adrenal mass. Ann Intern Med 1983; 98: 940.

19. OSELLA G, TERZOLO M, BORRETTA G, MAGRO G et al.: Endocrine evaluation of incidentally discovered adrenal mass. J Clin Endocrinol Metab 1994; 79: 1532-1539.

20. SEDDON JM, BARANETSKY N, VAN BOXEL PJ.: Adrenal incidentalomas. Need for surgery. Urology 1985; 25: 1-7.

Dr. F. Arredondo Martínez

C/ Ciudad de Aracena, 8 - 2 A

21001 Huelva

(Trabajo recibido el 25 abril de 2002) 